\title{
Mittelalterliche und frühneuzeitliche Inschriften in Württemberg
}

\author{
Neuere Ergebnisse der epigraphischen Forschung ${ }^{1}$ \\ Von Harald Drös
}

Die Inschriftenkunde oder Epigraphik des Mittelalters und der Neuzeit gehört heute unbestritten zum Kanon der Historischen Hilfswissenschaften. Allerdings hat sie sich zu einer recht eigentlichen wissenschaftlichen Disziplin neben den „Königsdisziplinen" Urkundenlehre und Paläographie und neben Siegelkunde, Heraldik, Genealogie, Münzkunde und Chronologie - um nur die wichtigsten zu nennen - erst etwa ab dem zweiten Drittel des vorigen Jahrhunderts entwickelt und gehört damit zu den vergleichsweise "jungen“"Wissenschaften. Gleichwohl hat man sich aber natürlich auch schon zuvor mit Inschriften beschäftigt, sie gesammelt, abgeschrieben oder abgezeichnet und auch publiziert. Eigene Regeln und Editionsrichtlinien, wie sie die antike Epigraphik schon lange kennt, gab es freilich nicht.

An älteren bedeutenderen Inschriftenveröffentlichungen für das württembergische Gebiet sind neben wenigen Einzelschriften ${ }^{2}$ zunächst die württembergischen Oberamtsbeschreibungen zu nennen ${ }^{3}$. In deren Bänden wurden Inschrif-

\footnotetext{
1 Der vorliegende Aufsatz beruht auf einem Vortrag, den ich am 10. Dezember 2011 beim Württembergischen Geschichts- und Altertumsverein in Stuttgart gehalten habe mit dem Titel „Zu Ehren und Gedächtnus ... Inschriften in Württemberg und ihre Erforschung“. Der erste Teil des Vortrags, der einen groben Überblick über die Erfassung und Erforschung der mittelalterlichen und frühneuzeitlichen Inschriften in Württemberg vom 16. Jahrhundert bis heute gab, wurde für die schriftliche Fassung stark gekürzt, während der zweite Teil, der einige neuere Ergebnisse der epigraphischen Forschung in den Blick nahm, überarbeitet und erweitert wurde.

${ }^{2}$ So beispielsweise Johann August Ferdinand Lenz: Sammlung sämmtlicher, noch vorhandener Epitaphien für die Stifts- und Hospital-Kirche zu Tübingen. Tübingen 1796; - Johann Friedrich Merckel (unter Mitarbeit von Johann Heinrich Tiedemann): Beschreibung der Fürstlichen Denkmale und Grabschriften in der Stiftskirche und der darinn befindlichen Gruft zu Stuttgardt, wie auch derer zu Tübingen und Ludwigsburg. Stuttgart 1798; - Albert Westermayer / Emil Wagner / Theodor Demmler: Die Grabdenkmäler der Stiftskirche zu St. Georg in Tübingen. Tübingen 1912.

${ }^{3}$ Beschreibung der württembergischen Oberämter. Hg. vom Königl. statistisch-topographischen Bureau, 64 Bde. Stuttgart 1824-1886, NF 7 Bde. 1893-1930.
} 
ten in zunehmendem Maße berücksichtigt, wenngleich die Textwiedergabe häufig nicht buchstabengetreu ist. Eine Gruppe von Inschriften, die im ausgehenden 19. und frühen 20. Jahrhundert das Interesse mehrerer Forscher fand, sind die Glockeninschriften. Besonders Theodor Schön hat in einer Reihe von zwischen 1898 und 1906 erschienenen Aufsätzen Inschriften württembergischer Glocken zusammengestellt - freilich zumeist nicht nach Autopsie, sondern vielfach gestützt auf die Texte der Oberamtsbeschreibungen und anderer Quellen. Eine solide Grundlage mit buchstabengetreuer und sehr zuverlässiger Textedition bietet dann erst der von Sigrid Thurm 1959 vorgelegte 1. Band des Deutschen Glockenatlas, der sämtliche Glocken in Württemberg und Hohenzollern berücksichtigt, allerdings beschränkt ist auf die noch erhaltenen Exemplare ${ }^{4}$.

Auch im Rahmen der württembergischen Kunstdenkmälerinventarisation fanden Inschriften gesteigert Beachtung. Während sie in den ältesten Bänden der Kunst- und Altertums-Denkmale noch recht stiefmütterlich behandelt wurden, bieten etwa die von Julius Baum, Hans Klaiber, Berthold Pfeiffer und Hans Christ bearbeiteten Bände des Donaukreises, erst recht die jüngeren Bände, reiches Inschriftenmaterial ${ }^{5}$. Die Textwiedergabe ist hier zumeist präziser und vollständiger als in den Oberamtsbeschreibungen.

An württembergischen Autoren, die für ihre Arbeiten in hohem Maße auch Inschriften herangezogen und gelegentlich sogar einzelnen Inschriften eigene Miszellen gewidmet haben, sind beispielsweise Ottmar Schönhuth (1806-64), der Mitbegründer des Vereins Württembergisch Franken, und Alfred Friedrich Klemm (1840-97) zu nennen. Besonders Klemms Studien zeichnen sich durch ein hervorragendes Gespür in inschriftenpaläographischen Fragen aus ${ }^{6}$.

Die meisten der genannten Werke beschränken sich im Wesentlichen auf das Sammeln und die reine Textwiedergabe von Inschriften. Dabei war in den wenigsten Fällen eine vollständige Erfassung der Inschriften einer bestimmten Region angestrebt, und von einer Erforschung der Inschriften als eigener Quellengattung kann - abgesehen von kleineren paläographisch orientierten Einzelmiszellen von Klemm und anderen - nicht die Rede sein. Die Epigraphik des Mittelalters und

${ }^{4}$ Deutscher Glockenatlas. Württemberg und Hohenzollern. Bearb. von Sigrid Thurm. München/Berlin 1959. Dort auf S. 713 Verzeichnis der Aufsätze Theodor Schöns.

${ }^{5}$ Die Kunst- und Altertums-Denkmale im Königreich Württemberg. Schwarzwaldkreis. 1897; Neckarkreis. 1889; Jagstkreis 1. 1907; Jagstkreis 2. 1913; Donaukreis 1. 1914; Die Kunst- und Altertums-Denkmale in Württemberg. Donaukreis 2. 1924; Oberamt Münsingen. 1926; Oberamt Ravensburg. 1931; Kreis Riedlingen. 1936. Fortsetzung: Die Kunstdenkmäler in Württemberg. Kreis Tettnang. 1937; Kreis Saulgau. 1938; Ehemaliger Kreis Waldsee. 1943; Ehemaliger Kreis Wangen. 1954; Ehemaliges Oberamt Künzelsau. 1962. Fortsetzung: Die Kunstdenkmäler in Baden-Württemberg. Ehemaliges Oberamt Ulm. 1978; Rems-Murr-Kreis. 2 Bde. 1983; Stadt Schwäbisch Gmünd. 4 Bde. 1995-2003.

${ }^{6}$ Vgl. z.B. Alfred Klemm: Die älteste Kircheninschrift unseres Landes. In: Korrespondenzblatt des Vereins für Kunst und Altertum in Ulm und Oberschwaben 2 (1877) S. 54-56; - Ders.: Inschrift an der Tür zu Künzelsau. In: WVjH 4 (1881) S. $150 f$. 
der Neuzeit als eigene Wissenschaftsdisziplin und die Erarbeitung von tragfähigen einheitlichen Editionsrichtlinien, die dem besonderen Quellencharakter von Inschriften gerecht werden, wurde, wie eingangs erwähnt, dann erst in den 30er Jahren des vorigen Jahrhunderts begründet und ihre Methoden in den folgenden Jahrzehnten entwickelt und verfeinert. Es war dies der Beginn des von dem Heidelberger Germanisten Friedrich Panzer initiierten und von den deutschen Akademien der Wissenschaften sowie der Österreichischen Akademie der Wissenschaften gemeinsam getragenen Forschungsunternehmens „Die Deutschen Inschriften“, das nunmehr seit fast 80 Jahren besteht. Ziel des Unternehmens ist die Sammlung, wissenschaftliche Bearbeitung und Veröffentlichung der mittelalterlichen und frühneuzeitlichen Inschriften des deutschen Sprachraums bis zur ungefähren Zeitgrenze $1650^{7}$.

Die ursprüngliche Sprengelverteilung zwischen den Akademien sah vor, dass Berlin (Preußische Akademie der Wissenschaften) für den Nordosten, die Göttinger Akademie für den Norden und Nordwesten und Leipzig (Sächsische Akademie der Wissenschaften) für die Mitte Deutschlands zuständig waren. München (Bayerische Akademie der Wissenschaften) erhielt neben Bayern auch Württemberg und Hohenzollern zugeteilt, während für die Heidelberger Akademie der gesamte Westen und Südwesten einschließlich Elsass und Lothringen und einschließlich Südhessen und der preußischen Rheinprovinz vorgesehen war. Der Wiener $\mathrm{Zu}$ ständigkeitsbereich umfasste neben Österreich auch Südtirol.

Danach wurde vor allem in Heidelberg und Wien mit der praktischen Arbeit begonnen. In den folgenden Jahren einigte man sich auf den Titel „Die Deutschen Inschriften“ (DI) für die zu publizierenden Inschriftenbände, und es wurde eine Unterteilung nach Akademieserien festgelegt, der zu Folge beispielsweise die von der Heidelberger Akademie erarbeiteten Bände in der „Heidelberger Reihe“, die der Bayerischen Akademie in der „Münchener Reihe“ erscheinen sollten. Zwar konnte noch während des Zweiten Weltkriegs 1942 der erste Band der Gesamtreihe und Band 1 der Heidelberger Reihe mit den „Inschriften des badischen Main- und Taubergrundes" herausgebracht werden ${ }^{8}$, doch kam das junge Unternehmen durch

7 Vgl. zuletzt Harald Drös: Deutsche Inschriften. In: Die Forschungsvorhaben der Heidelberger Akademie der Wissenschaften 1909-2009. 100 Jahre Heidelberger Akademie der Wissenschaften. Hg. von Volker Sellin, Eike Wolgast u. Sebastian Zwies. Heidelberg 2009. S. 113-119. - Ferner ausführlich Renate Neumüllers-Klauser: Die Inschriftensammlung der Heidelberger Akademie der Wissenschaften. In: Heidelberger Jahrbücher 10 (1966) S. $113-$ 134. - Zum Gesamtunternehmen vgl. Walter Koch: 50 Jahre Deutsches Inschriftenwerk (1934-1984). In: Deutsche Inschriften. Fachtagung für mittelalterliche und neuzeitliche Epigraphik Lüneburg 1984. Vorträge und Berichte. Hg. von Karl Stackmann (Abhandlungen der Akademie der Wissenschaften in Göttingen, Phil.-Hist. Klasse, 3. Folge Nr. 151). Göttingen 1986. S. 15-45.

${ }^{8}$ Die Inschriften des badischen Main- und Taubergrundes. Wertheim-Tauberbischofsheim. Ges.u. bearb. von Ernst Cucuel u. Hermann Eckert, Vorwort zum Gesamtwerk von Friedrich Panzer (DI 1). Stuttgart 1942, ND 1969. 
den Krieg fast ganz zum Erliegen, und nach Kriegsende bedurfte es einer langen Anlaufphase, bis die Arbeiten wieder in Gang kamen.

Nach der ursprünglichen Sprengelaufteilung war also, wie gesehen, für Württemberg die Bayerische Akademie der Wissenschaften zuständig. Angesichts der erdrückend großen Masse des inschriftlichen Materials, wie es in Bayern vorhanden ist - erwähnt seien nur die Städte Regensburg, Augsburg, Nürnberg, aber auch Bamberg oder Passau mit ihren riesigen Beständen -, und angesichts der ungenügenden Personalausstattung kann es nicht verwundern, dass sich die Bayerische Akademie um Württemberg wenig - um nicht zu sagen: gar nicht - kümmerte. Kurioserweise ist aber als erster Band der Münchener Reihe (in der Zählung aber als zweiter) und als vierter Band der Gesamtreihe ausgerechnet ein württembergisches Bändchen erschienen: 1958 kamen die von Fritz Viktor Arens erarbeiteten „Inschriften der Stadt Wimpfen am Neckar“ heraus9. Dies bedarf einer Erklärung. Der Kunsthistoriker Arens hatte bereits den voluminösen Band der Mainzer Inschriften in den Kriegsjahren und danach bearbeitet, der in der Heidelberger Reihe 1951-55 in Teillieferungen gedruckt worden und 1958 erschienen war ${ }^{10}$. Schon 1943 hatte Arens parallel dazu mit der Sammlung der Inschriften von Wimpfen begonnen, das ja bis 1945 zum Land Hessen gehörte. Nach dem Krieg wurde die Stadt dann zunächst dem badischen Landkreis Sinsheim angegliedert, 1951 aber schließlich dem württembergischen Landkreis Heilbronn zugeschlagen. Somit fiel Wimpfen in den Zuständigkeitsbereich der Bayerischen Akademie, und als die Sammlung fertig vorlag, entschloss man sich in Absprache mit der Heidelberger Akademie, den Band in die Münchener Reihe aufzunehmen. Einem Wunsch des Bearbeiters entsprechend umfasst der Band die Inschriften bis zum Jahr 1800.

Damit war zwar eigentlich ein Anfang der „Deutschen Inschriften“ in Württemberg gemacht, doch blieb es auch dabei. München blieb verständlicherweise bei seiner Konzentration auf Bayern, als nächster Band der Münchener Reihe, der die Bandzählung 1 erhielt, erschien noch im selben Jahr 1958 zum Münchener Stadtjubiläum der Band mit den Inschriften von Stadt und Landkreis München ${ }^{11}$. In den folgenden Jahren wurden lediglich durch einen freien Mitarbeiter zaghafte Versuche unternommen, die Inschriften in der Stadt Ulm aufzunehmen. Über Fotoaufnahmen gingen diese Ansätze allerdings nicht hinaus. Weitere Vorarbeiten betrafen Ellwangen. Inschriftenbände wurden daraus gleichwohl nie. Die Ellwanger Inschriften wurden immerhin schließlich von Klaus-Ulrich Högg in zwei Jahrgängen des Ellwanger Jahrbuchs 1984 und 1985 ediert $^{12}$.

9 Die Inschriften der Stadt Wimpfen am Neckar. Ges.u. bearb. von Fritz Viktor Arens (DI 4). Stuttgart 1958.

10 Die Inschriften der Stadt Mainz von frühmittelalterlicher Zeit bis 1650. Ges. u. bearb. von Fritz Viktor Arens auf Grund der Vorarbeiten von Konrad F. Bauer (DI 2). Stuttgart 1958.

${ }_{11}$ Die Inschriften der Stadt und des Landkreises München. Ges.u. bearb. von Rudolf M. Kloos (DI 5). Stuttgart 1958.

12 Klaus-Ulrich Högg: Die Inschriften im Kreuzgang der ehem. Stiftskirche St. Vitus zu 
Angesichts dieser unbefriedigenden Situation, dass Württemberg innerhalb des Deutschen Inschriftenwerks quasi unberücksichtigt blieb, hat Hansmartin Decker-Hauff die Initiative ergriffen und einige seiner Schüler mit Inschriftensammlungen nach dem Muster der DI in Altwürttemberg betraut. Daraus resultierte eine Reihe von Dissertationen ${ }^{13}$.

Die gerade skizzierte Situation änderte sich grundlegend 1970/71. Die deutsche Akademienlandschaft hatte sich inzwischen verändert durch die Neugründung der Akademie der Wissenschaften und der Literatur zu Mainz 1949 und der Rheinisch-Westfälischen Akademie der Wissenschaften (heute: Nordrhein-Westfälische Akademie der Wissenschaften und Künste) zu Düsseldorf 1970. Beide Akademien erklärten sich zur Mitarbeit an den DI bereit, so dass es - auch im Zuge einer Neuorganisation der interakademischen Zusammenarbeit und der Installierung des sog. Akademienprogramms zur finanziellen Absicherung der Forschungsprojekte durch je hälftige Bund-Länderfinanzierung - zu einer Neuverteilung der Sprengel zwischen den DI-Arbeitsstellen kam. Seit 1971 ist nunmehr Heidelberg zuständig für das gesamte Bundesland Baden-Württemberg ${ }^{14}$.

In Heidelberg war man bislang auf die Erfassung badischer Bestände fokussiert gewesen, möglichst in der Fläche kontinuierlich fortschreitend von Norden nach Süden. Erschienen waren die Bände Badischer Main- und Taubergrund, Mosbach-Buchen (einschließlich dem bayerischen Kreis Miltenberg), Stadt und (alter) Landkreis Heidelberg und nach Abschluss der Kreis- und Gemeindereform die

Ellwangen bis zur Säkularisation der Fürstpropstei. In: Ellwanger Jahrbuch 30 (1983/84) S. 131-212;-Ders.: Die Inschriften in der ehemaligen Stiftskirche St. Vitus zu Ellwangen bis zur Säkularisation der Fürstpropstei. In: Ellwanger Jahrbuch 31 (1985) S. 158-202.

${ }^{13}$ Klaus Autbert Maier: Die Inschriften des Landkreises Saulgau. Diss. Tübingen 1970; - Ottfried Hauser: Die Inschriften der Kreise Münsingen und Ehingen. Diss. Tübingen 1972; - Dietmar-H. Voges: Die deutschen Inschriften in Stadt und Kreis Reutlingen. Diss. Tübingen 1974 (Göppinger Akademische Beiträge 92). Göppingen 1975. Hinzu kommen die Zulassungsarbeiten von Hans-Ulrich Schäfer über den alten Landkreis Ludwigsburg (begonnen 1965) und von Dieter Reichert über die Inschriften des ehemaligen Oberamts Schorndorf (1967). Mit Ausnahme der beiden letzteren Arbeiten mündeten diese Sammlungen jedoch nicht in spätere DI-Bände.

${ }^{14}$ Nicht unerwähnt darf bleiben, dass sich die Sächsische und die Berliner Akademie auch nach Gründung der DDR zunächst noch am Gesamtunternehmen beteiligten, so dass zwischen 1959 und 1967 insgesamt vier Bände der Berliner Reihe erscheinen konnten, doch kurz danach wurde die Arbeit aus politischen Gründen dort faktisch eingestellt. Erst nach dem Fall der Mauer und der deutschen Wiedervereinigung konnte in der nunmehrigen Berlin-Brandenburgischen Akademie im Jahr 1992 eine Forschungsstelle neu etabliert werden; 1996 folgte die Sächsische Akademie mit der Errichtung einer Arbeitsstelle in Halle. Dass nach nur wenigen Jahren die Berliner Akademie wieder aus dem Unternehmen ausstieg, ist äußerst bedauerlich. Immerhin konnte aber für Mecklenburg-Vorpommern eine Filiale der Göttinger Akademie in Greifswald zumindest einen Teil des Berliner Zuständigkeitsbereichs übernehmen. Derzeit sind somit an dem Vorhaben sieben Akademien mit acht Forschungsstellen beteiligt. 
noch während der Durchführung der Reform begonnenen Bände Rhein-NeckarKreis II und Karlsruhe ${ }^{15}$.

Sofort nach der Neuverteilung der Zuständigkeiten entschied man sich dafür, die Arbeit nunmehr auch in Württemberg in Angriff zu nehmen. Zum einen begann Renate Neumüllers-Klauser mit der Erfassung der Inschriften im Enzkreis, der zumindest teilweise württembergisches Gebiet betraf, und zum anderen wurde, aufbauend auf die Zulassungsarbeit von Hans-Ulrich Schäfer, der Landkreis Ludwigsburg ins Programm aufgenommen. Die Materialsammlung von Schäfer, die den Altkreis Ludwigsburg umfasste, wurde durch Anneliese Seeliger-Zeiss um die Inschriften der Gebiete ergänzt, die durch die Kreisreform zum neu zugeschnittenen Landkreis hinzugekommen waren. Mit den Inschriften des Enzkreises und mit den Inschriften des Landkreises Ludwigsburg konnten somit 1983 und 1986 die ersten württembergischen Inschriftenbestände in der Heidelberger Reihe der DI erscheinen ${ }^{16}$.

Seither hat sich die Heidelberger Arbeitsstelle bemüht, den württembergischen Landesteil etwas stärker zu berücksichtigen, um den „badischen Vorsprung“ aufzuholen. Insgesamt sollten badischer und württembergischer Landesteil etwa gleich starke Berücksichtigung finden. Zum „Aufholprogramm“ gehörten die württembergischen Landkreise Calw (ersch. 1992), Rems-Murr-Kreis (ersch. 1994), dessen Band sich zum Teil auf Vorarbeiten einer älteren Zulassungsarbeit stützen konnte und in großen Teilen von dem ehrenamtlichen Mitarbeiter Gerhard Fritz erarbeitet wurde, sowie Göppingen (ersch. 1997) und Böblingen (ersch. 1999) ${ }^{17}$.

Die Editionsarbeit wird im Regierungsbezirk Karlsruhe demnächst abgeschlossen sein. Mit dem letzten noch ausstehenden Landkreis Freudenstadt, der derzeit von Ilas Bartusch bearbeitet wird und voraussichtlich 2014 zum Abschluss kommt, ist auch hier fast ausschließlich ehemals württembergisches Gebiet betroffen. Im

15 Die Inschriften der Landkreise Mosbach, Buchen und Miltenberg. Auf Grund der Vorarbeiten von Ernst Cucuel ges. u. bearb. von Heinrich Köllenberger (DI 8). Stuttgart 1964; - Die Inschriften der Stadt und des Landkreises Heidelberg. Ges. u. bearb. von Renate Neumüllers-Klauser (DI 12). Stuttgart 1970; - Die Inschriften des Rhein-Neckar-Kreises (II). Ehemaliger Landkreis Mannheim, Ehemaliger Landkreis Sinsheim (nördlicher Teil). Ges. u. bearb. von Renate Neumüllers-Klauser unter Mitarbeit von Anneliese Seeliger-Zeiss (DI 16). München 1977; - Die Inschriften des Großkreises Karlsruhe. Ges. u. bearb. von Anneliese Seeliger-Zeiss (DI 20). München 1981.

16 Die Inschriften des Enzkreises bis 1650. Bearb. von Renate Neumüllers-Klauser (DI 22). München 1983; - Die Inschriften des Landkreises Ludwigsburg. Ges. u. bearb. von Anneliese Seeliger-Zeiss u. Hans Ulrich Schäfer (DI 25). Wiesbaden 1986.

17 Die Inschriften des Landkreises Calw. Ges.u. bearb. von Renate Neumüllers-Klauser (DI 30). Wiesbaden 1992; - Die Inschriften des Rems-Murr-Kreises. Ges. u. bearb. von Harald Drös u. Gerhard Fritz unter Benutzung der Vorarbeiten von Dieter Reichert (DI 37). Wiesbaden 1994; - Die Inschriften des Landkreises Göppingen. Ges.u. bearb. von Harald Drös (DI 41). Wiesbaden 1996; - Die Inschriften des Landkreises Böblingen. Ges. u. bearb. von Anneliese Seeliger-Zeiss (DI 47). Wiesbaden 1999. 
Regierungsbezirk Stuttgart konzentriert sich die Inschriftenerfassung derzeit auf die sehr inschriftenreiche Region Württembergisch Franken. An den Altkreis Mergentheim schloss sich der Hohenlohekreis an, der mit seinem unerwartet großen Inschriftenbestand erstmals innerhalb der Heidelberger Reihe eine zweibändige Edition erforderte ${ }^{18}$. Seit 2009 werden nun die Inschriften des Landkreises Schwäbisch Hall erfasst, wobei hier aufgrund der Größe von vorneherein eine Zweiteilung in Altkreis Crailsheim und den Südwesten des Kreisgebiets nötig war.

Die weitere Planung sieht eine möglichst vollständige Erfassung der Inschriften des Regierungsbezirks Stuttgart vor. Nachdem 2012 für das Gesamtprojekt im Rahmen des Akademienprogramms jetzt das Jahr 2030 als Laufzeitende festgesetzt wurde, muss die Heidelberger Arbeitsstelle notgedrungen auf eine Fortführung der eigentlich geplanten Arbeiten im badischen Landesteil, also im Regierungsbezirk Freiburg, verzichten zugunsten eines Abschlusses zumindest im Regierungsbezirk Stuttgart und damit in der Nordhälfte des Landes. Im Süden wird zunächst der Landkreis Esslingen, dann Stuttgart bearbeitet, und an Schwäbisch Hall sollen sich Landkreis und Stadt Heilbronn anschließen, und schließlich soll, wenn möglich, die Region Ostwürttemberg (Ostalbkreis und Lkr. Heidenheim) den Abschluss bilden.

Zum Konzept der Heidelberger Arbeitsstelle gehört seit 1989 eine landesweite Fotoinventarisierung der Inschriftendenkmäler als Vorarbeit für künftige Editionen ${ }^{19}$. Die Dringlichkeit einer solchen Vorarbeit ist - gerade angesichts der drohenden zunehmenden Verwitterung von im Freien befindlichen Inschriften unmittelbar einsichtig. Durch die jetzt absehbare Beschränkung der Edition auf die Nordhälfte des Landes wird der Fotoinventarisierung freilich der Charakter einer Vorarbeit genommen. Immerhin wird aber nach Abschluss der Fotokampagne in einem zweiten noch ausstehenden Durchgang auch für den Süden des Landes zumindest ein Fotoarchiv für künftige epigraphische Forschung zur Verfügung stehen.

Nicht übergehen möchte ich, dass in den letzten Jahren auch außerhalb der DI-Reihe einige epigraphische Arbeiten erschienen sind, die württembergische Bestände betreffen. Von den umfangreicheren verdienen hier in erster Linie Erwähnung die 1989 erschienenen „Grabdenkmale im Kloster Bebenhausen“, eine Edition, die sich weitgehend an den DI-Richtlinien orientiert ${ }^{20}$. Dann im Rahmen der Großprojekte des Landesdenkmalamts zu den Klöstern Maulbronn und Alpirsbach die Edition der Maulbronner Inschriften aus der Zeit nach 1650 und die Edi-

${ }_{18}$ Die Inschriften des ehemaligen Landkreises Mergentheim. Ges.u. bearb. von Harald Drös (DI 54). Wiesbaden 2002; - Die Inschriften des Hohenlohekreises. Ges. u. bearb. von Harald Drös (DI 73). Wiesbaden 2008.

19 Anneliese Seeliger-Zeiss: Historische Grabmäler in Baden-Württemberg. Inventarisierung als Instrument gegen den Verlust von Kirchengut. In: ZWLG 54 (1995) S. 379-392.

${ }^{20}$ Die Grabdenkmale im Kloster Bebenhausen. Bearb. von Hans Gerhard Brand, Hubert Krins und Siegwalt Schiek (Beiträge zur Tübinger Geschichte 2). Stuttgart 1989. 
tion der Inschriften des Klosters Alpirsbach ${ }^{21}$. Ferner in der Zeitschrift Württembergisch Franken 2005 und 2007 die Dokumentation der Grabmäler in der Haller Urbanskirche und der Katharinenkirche durch Hans Werner Hönes ${ }^{22}$ sowie die „Grabmonumente der Stiftskirche in Tübingen“" von Stefanie Knöll 2007²3.

Im Folgenden sollen anhand einiger Beispiele Ergebnisse der epigraphischen Forschung der letzten Jahre in Württemberg vorgestellt werden. Aufgrund des derzeitigen Arbeitsschwerpunkts liegt das Hauptaugenmerk dabei zwangsläufig auf Württembergisch Franken. Eine Zusammenfassung der wichtigsten Ergebnisse, die sich aus der epigraphischen Bearbeitung eines jeden neuen Landkreises ergeben, findet sich in der Regel in den Einleitungskapiteln des betreffenden DIBandes. Die Rezeption dieser Einzelergebnisse durch die Forschung ist allerdings recht unbefriedigend. Daher mag es durchaus nützlich und angebracht sein, einige dieser Resultate hier im Überblick zu wiederholen und so einem weiteren Kreis zur Kenntnis zu bringen.

Einen Schwerpunkt der Arbeit der Heidelberger Forschungsstelle bildete stets die Inschriftenpaläographie, d.h. die Untersuchung der epigraphischen Schriftformen und ihrer Entwicklung, als Beitrag zur allgemeinen Schriftgeschichte. Gerade der flächendeckende Ansatz, die lückenlose Erfassung großer zusammenhängender Gebiete einschließlich der ländlichen Regionen abseits der kulturellen Zentren eröffnet die Chance, Werkstattzusammenhänge aufzuspüren, konkret: anhand von Schriftgemeinsamkeiten einzelne Schriftdenkmäler bestimmten bekannten oder anonymen Werkstätten oder Meistern zuzuordnen und deren Absatzgebiet zu rekonstruieren. Dass dies aufgrund der schlechten Überlieferungssituation immer nur ansatzweise gelingen kann, liegt auf der Hand. Gelegentlich lassen sich aber doch imposante Werkgruppen zusammenstellen und mitunter auch mit konkreten Meisternamen verbinden. Es sind dies nicht unbedingt immer

${ }^{21}$ Georg u. Renate Vogeler: Manibus sacrum. Die Grabinschriften im Kloster Maulbronn als „Denk-Male“ für 150 Jahre Klostergeschichte von 1656 bis 1807. In: Maulbronn. Zur 850jährigen Geschichte des Zisterzienserklosters. Hg. vom Landesdenkmalamt Baden-Württemberg (Forschungen und Berichte der Bau- und Kunstdenkmalpflege in Baden-Württemberg 7). Textbd. Stuttgart 1997. S. 491-529; - Anneliese Seeliger-Zeiss, Die Inschriften. In: Alpirsbach. Zur Geschichte von Kloster und Stadt (Forschungen und Berichte der Bau- und Kunstdenkmalpflege in Baden-Württemberg 10). Textbd. 1. Stuttgart 2001, S. 515-588.

${ }^{22}$ Hans Werner Hönes: Urbanskirche Schwäbisch Hall. Dokumentation der Epitaphe und Grabmale. In: Württembergisch Franken 89 (2005) S. 169-219; - Ders.: Katharinenkirche Schwäbisch Hall. Epitaphe, Grabmale, Tafelgemälde, Gedenk- und Stiftertafeln. In: Württembergisch Franken 90/91 (2006/2007) S. 227-278.

${ }^{23}$ Stefanie A. Knöll: Die Grabmonumente der Stiftskirche in Tübingen (Beiträge zur Tübinger Geschichte 13). Stuttgart 2007. 
die kunsthistorisch bedeutendsten Werke, aber gerade für die Erforschung der Alltagsgeschichte kommt der Ermittlung von Werkstätten zweiter oder dritter Kategorie und ihrer Tätigkeit eine nicht zu unterschätzende Bedeutung zu, zumal sich die kunsthistorische Forschung dieser Werke in der Regel nicht annimmt.

Bei der Bearbeitung der Inschriften des ehemaligen Landkreises Mergentheim rückte mit Michel Niklas von Reinsbronn ein bisher nur wenig beachteter Steinmetz und Bildhauer ins Blickfeld. Seine Lebensdaten sind nicht bekannt. Er wurde wohl um die Mitte des 16. Jahrhunderts geboren und scheint bald nach 1612 gestorben zu sein. Seine Werkstatt hatte er in Reinsbronn (Stadt Creglingen, Main-Tauber-Kreis), doch stammt er vermutlich aus Weikersheim ${ }^{24}$. Um 1601 verlegte er dann seine Werkstatt nach Enheim (Lkr. Kitzingen) ${ }^{25}$. Für seine Inschriften, vorweg auf Grabmälern, aber auch an Altären, Kanzeln und Taufsteinen, verwendete Niklas sowohl Kapitalis als auch Fraktur, häufig auch beide Schriftarten auf einem Inschriftenträger kombiniert. Seine sehr prägnante Kapitalis lässt sich allein im Main-Tauber-Kreis auf insgesamt 16 Inschriftenträgern aus dem Zeitraum von 1575 bis 1608 nachweisen. Die auffälligsten Merkmale dieser sehr regelmäßigen Schrift sind die stark gekrümmten oder s-förmig geschwungenen Sporen an allen freien Bogenenden und die konsequente rechtsschräge Ausrichtung sämtlicher Balkensporen ${ }^{26}$ (Abb.1). Ebenso markant ist Niklas' Fraktur, die sich im Altkreis Mergentheim auf 21 Werken zwischen 1580 und 1612 findet, die aber bei den Gemeinen eine gewisse Entwicklung über die Jahre hinweg hin zu einer zunehmenden Ausrundung der Bögen erkennen lässt ${ }^{27}$. Ihr Hauptmerkmal sind die ausgewogenen, zumeist schmucklosen und nur ungewöhnlich wenige Doppelformen aufweisenden Versalien, die gleichwohl dadurch sehr variabel sind, dass sie durch mehr oder minder weit nach links ausholende, meist s-förmige Anschwünge erheblich verbreitert sein können und somit zugleich als Zeilenfüller dienen, ohne das harmonische Schriftbild zu stören.

Außerhalb des Altkreises Mergentheim können dem Oeuvre Niklas' allein anhand des Schriftvergleichs in Baden-Württemberg zwei weitere und in Unterfranken mindestens zehn Inschriftendenkmäler zugewiesen werden. Niklas' Sohn, der 1617 einen Bildstock in Igersheim mit $P N B$ signierte und dessen Name inzwischen als Philipp identifiziert werden konnte (also: Philipp Niklas Bildhauer) ${ }^{28}$, führte die Werkstatt seines Vaters fort. Er bediente sich fast derselben Kapitalis, wohingegen er die Fraktur in charakteristischer Weise abwandelte und deutlich runder und

${ }^{24}$ Diesen Hinweis sowie zahlreiche weitere Informationen zu Leben und Werk verdanke ich Pfarrer Dr. Hans-Ulrich Hofmann, Marktbreit-Gnodstadt.

25 Auch diesen Hinweis verdanke ich Dr. Hans-Ulrich Hofmann.

${ }^{26}$ Ausführliche Beschreibung der Schriftmerkmale in DI 54 (Mergentheim, wie Anm. 18) S. LXIf.

${ }^{27}$ Ebd. S. LXVf.

28 Ebd. S. LXII, Nr. 415, hatte ich noch Peter als Vornamen erwogen. Philipp Niklas ist jedoch archivalisch ab 1608 gut bezeugt, so etwa Gemeindearchiv Enheim Bd.1, fol. 260. Freundliche Mitteilung von Dr. Hans-Ulrich Hofmann. 


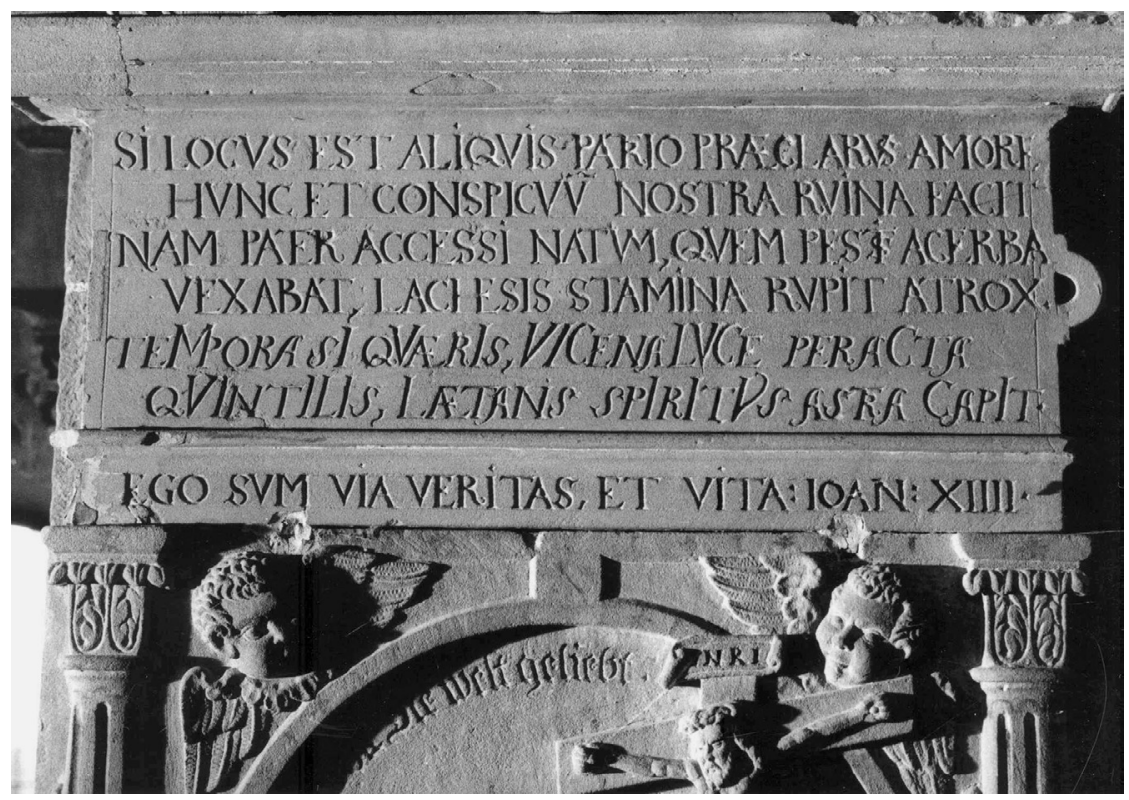

Abb. 1: Michel Niklas, Epitaph des Matthäus Lilienfein (1584) in Elpersheim (Stadt Weikersheim, Main-Tauber-Kreis).

im Mittellängenbereich gedrungener gestaltete (Abb.2). Seine Frakturversalien besitzen teilweise ähnliche s-förmig geschwungene Sporen wie sie an den freien Bogenenden der Kapitalis zu beobachten sind. Insgesamt neun zwischen 1607 und 1635 entstandene Inschriftenträger können dem jüngeren Niklas im Altkreis Mergentheim zugewiesen werden, mittlerweile mindestens ein Dutzend weitere im angrenzenden Unterfranken. Die beiden Niklas waren sowohl für den Niederadel als auch für die bürgerliche Führungsschicht tätig.

Wesentlich prominenter und besser erforscht ist die über vier Generationen im 17. Jahrhundert in Forchtenberg ansässige und tätige Steinmetz- und Bildhauerfamilie $\mathrm{Kern}^{29}$, von der für die Inschriftenproduktion vor allem Michael Kern II. $(† 1634)$, sein Sohn Michael III. $(† 1649)$ und dessen Sohn Achilles $(† 1691)$ in Frage kommen. Ihre Schriften - Kapitalis und Fraktur - sind weit weniger charakteristisch als die der beiden Niklas ${ }^{30}$, und sie lassen sich nicht immer eindeutig von an-

${ }^{29}$ Die Künstlerfamilie Kern 1529-1691. Hohenloher Bildhauer und Baumeister des Barock. Hg. von der Stadt Forchtenberg anläßlich des 700-jährigen Stadtjubiläums, Sigmaringen 1998; - Vera Schneider: Michael Kern (1580-1649). Leben und Werk eines deutschen Bildhauers zwischen Renaissance und Barock (Forschungen aus Württembergisch Franken 49). Ostfildern 2003.

30 Vgl. DI 73 (Hohenlohekreis, wie Anm. 18) S. 70-73, 77-79. 


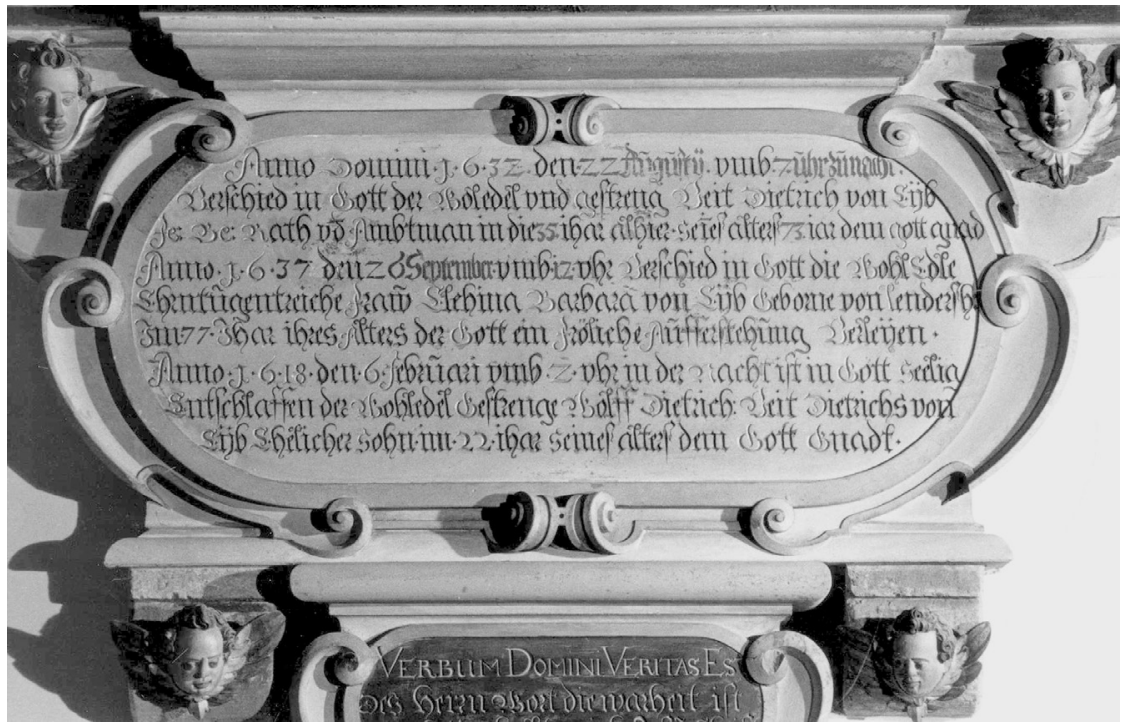

Abb. 2: Philipp Niklas, Epitaph für Veit Dietrich, Elichina Barbara und Wolf Dietrich von Eyb (1628) in Creglingen (Main-Tauber-Kreis).

deren im Hohenlohischen tätigen Werkstätten unterscheiden, zumal es zum Austausch von Personal zwischen den Werkstätten gekommem zu sein scheint, so unter anderem mit der des Philipp Kolb in Öhringen ${ }^{31}$. Auch der Bruder Michaels III., der gräflich hohenlohische Baumeister Georg Kern († vor 1643), bediente sich einer ähnlichen Schrift. Hier bedarf es wohl noch eingehenderer Studien als sie im Rahmen der DI-Bände geleistet werden können, um die Werkstattzusammenhänge näher aufzuhellen.

Immerhin lässt sich das Oeuvre von Michael Kern II. und III. durch den Schriftvergleich, ergänzend auch durch den viel zu selten von kunsthistorischer Seite berücksichtigten heraldischen Stilvergleich, jetzt erheblich erweitern. Den prominenten Arbeiten für gräfliche Auftraggeber (Hohenlohe, Oettingen, Wertheim, Schenken von Erbach), die Würzburger Fürstbischöfe oder das Kloster Schöntal können nun etliche einfachere Arbeiten, vorweg für den Niederadel, angereiht werden. Besonders ergiebig war die inschriftenpaläographische Untersuchung jedoch für den letzten der Kern-Dynastie, den erst 1691 verstorbenen Achilles Kern. Er bediente sich einer dünnstrichigen, relativ schmal proportionierten Kapitalis, die er auch in einer schrägliegenden Version verwendete. Markantester Einzelbuchstabe, weil innerhalb der Kapitalis eine Fremdform, ist ein zweibogiges $E$.

${ }^{31} \mathrm{Zu}$ diesem vgl. ebd. S. $70 \mathrm{f}$. 


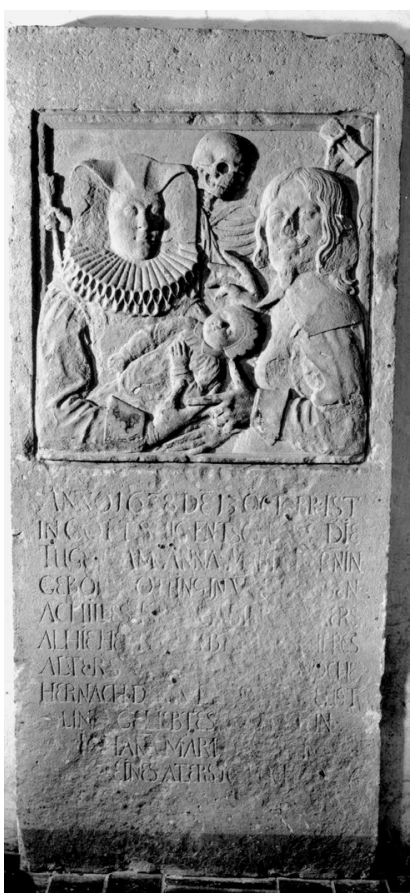

Abb. 3: Achilles Kern, Grabmal für Anna Maria und Johanna Maria Kern (1638) in Forchtenberg (Hohenlohekreis).

Dem Achilles können aufgrund dieser Schrift acht zwischen 1633 und 1650 entstandene Inschriftendenkmäler im Hohenlohekreis und noch wesentlich mehr Werke aus der zweiten Jahrhunderthälfte zugewiesen werden, darunter auch das Forchtenberger Grabmal für seine Frau und seine Tochter von 1638, auf dem sich Achilles in einem Selbstbildnis verewigt hat ${ }^{32}$ (Abb. 3), was erst im Zuge der Inschriftenbearbeitung erkannt wurde.

Ein weiteres, eher unscheinbares Inschriftendenkmal, das erst nach Erscheinen des Hohenlohekreis-Bandes zum Vorschein kam und dessen Inschriftenedition deshalb hier nachgetragen wird, kann aufgrund seiner Schriftformen ebenfalls der Kern-Werkstatt, näherhin Michael II., zugewiesen werden. Es handelt sich bei diesem Neufund um das Fragment einer Grabplatte oder eines Epitaphs für den 1605 verstorbenen Jörg Zöller, Bürger zu Forchtenberg. Es wurde vor wenigen Jahren außen an der Südwand der Forchtenberger Friedhofskapelle angebracht (Abb. 4). Der ursprüngliche Standort auf dem Friedhof ist nicht bekannt. Bei den Inschriftenaufnahmearbeiten 2003 war mir das Grabmal verborgen geblieben, vermutlich

32 Ebd. Nr. 849. 


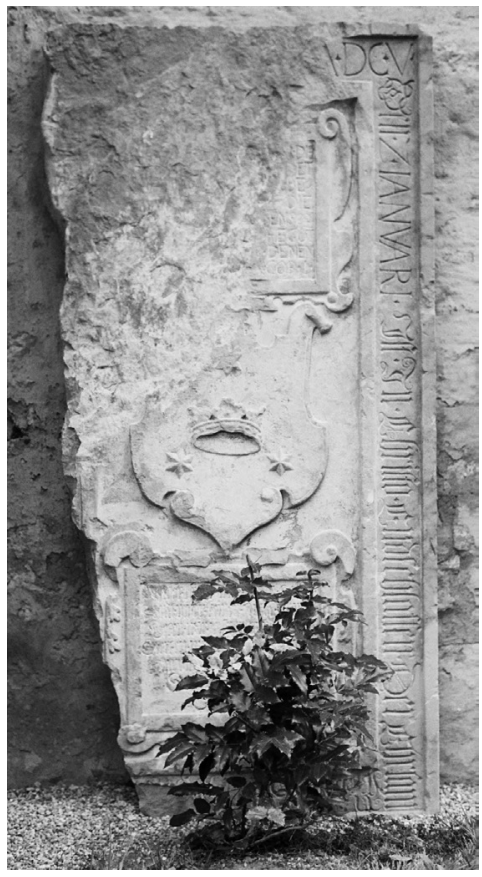

Abb. 4: Michael Kern II., Grabmal des Jörg Zöller (1605) in Forchtenberg (Hohenlohekreis).

war es einer der völlig eingesunkenen und zugewucherten Steine, die östlich der Kapelle im Boden lagen ${ }^{33}$. Die hochrechteckige Platte aus hellem Sandstein ist mit einem zwischen Rahmenleisten umlaufenden Sterbevermerk (A) versehen. Im Feld befindet sich zentral ein reliefierter Wappenschild (Krone, begleitet von drei 1:2 gestellten sechsstrahligen Sternen), darüber und darunter je eine rechteckige Kartusche mit Bibelspruch: oben (B), unten (C). Der linke Rand der Platte ist weggebrochen, die Oberfläche ist im linken oberen Drittel völlig abgewittert. Erheblicher Schriftverlust ist vor allem bei den Inschriften (A) und (B) eingetreten; Inschrift (C) ist durch Verwitterung beeinträchtigt. Als Schriftarten sind Fraktur und Kapitalis eingesetzt.

33 Ein weiteres Epitaph, das der 1609 verstorbenen Barbara Müller, - sehr wahrscheinlich ebenfalls ein Werk des Michael Kern II. - war 1990 noch in die westliche Friedhofsmauer eingelassen, lag zur Zeit der Inschriftenerfassung 2003 dann aber provisorisch am Boden, war teilweise im Erdreich versunken und musste bei den Aufnahmearbeiten erst mühsam ausgegraben werden. Vgl. DI 73 (Hohenlohekreis, wie Anm. 18) Nr. 624. Auch dieses Grabmal wurde mittlerweile an der Südwand der Friedhofskapelle angebracht. 
A $\quad[---] \mathrm{M}^{\mathrm{a})} \cdot \mathrm{DC} \cdot \mathrm{V} \cdot / \mathrm{Den} \cdot 2 \cdot \mathrm{IANVARI} \cdot \mathrm{Jst} \cdot \mathrm{Jn} \cdot$ Christo $\cdot$ Endschlaffen $\cdot$ Der Ehrsam / Jörg $\cdot$ Zoller $\cdot$ B [urger ..... / - - ]

B [SELIG IST DER MAN DE]R ${ }^{\mathrm{b})} \cdot \mathrm{DIE} /$ [ANFECHTVNG ERDVL]DET / [DENN NACHDEM ER] BE=/[WERET IST WIRD ER] DIE / [KRONE DES L]EBENS c) / [EMPFAHEN WELC]HE · GOT / [VERHEISSEN] $\mathrm{HAT} \cdot \mathrm{DENEN} /\left[\mathrm{DIE}\right.$ IN LIEBHABEN $\left.{ }^{34} \cdot \mathrm{IA}\right] \mathrm{COB} \cdot \mathrm{I} \cdot$

C Vnser $\cdot$ keiner $\cdot$ lebtt $\cdot \mathrm{Jhm} \cdot$ Selber $\cdot /$ Vnd $\cdot$ vnser $\cdot$ keiner $\cdot$ stirbt $\cdot$ ihm $\cdot$ selber $^{\text {d) }}$ / Leben · wir $\cdot$ so $\cdot$ leben $\cdot$ wir $\cdot$ dem $\cdot$ hern / Sterben · wir $\cdot$ so $\cdot$ sterben $\cdot$ wir $\cdot$ dem $\cdot$ hern $^{\text {e) }} /$ Darvm $\cdot$ wir $\cdot$ leben $\cdot$ oder $\cdot$ sterben / So $\cdot$ sein $\cdot$ wir $\cdot$ dem $\cdot$ hernn ${ }^{35} \cdot /$ Rom $\cdot 14$.

a) Vom $M$ nur der schräge rechte Schaft erhalten. - b) Ergänzung - wie auch im Folgenden nach dem Text der Lutherbibel. Vom $R$ nur der rechte Teil des Bogens und ein Teil der Cauda erhalten. - c) Danach ein Zierschnörkel als Zeilenfüller. - d) er außerhalb des Schriftfelds auf der erhabenen Rahmenleiste. - e) $n$ außerhalb des Schriftfelds auf der erhabenen Rahmenleiste.

Die Fraktur ist im Mittellängenbereich äußerst schmal proportioniert. Die Buchstaben $b$ und $t$ sind ganz in das Mittelband eingefügt und bilden keine Oberlänge aus. Die Versalien sind reich verziert, bisweilen aber etwas unförmig. Auch die dünnstrichige Kapitalis weist schmale Proportionen auf. $E$ hat einen ausgesprochen kurzen Mittelbalken; die Schäfte des $M$ sind schräggestellt. Als Worttrenner dienen mächtige Quadrangel auf halber Zeilenhöhe. Den Schriftmerkmalen zufolge wurde das Grabmal, wie erwähnt, zweifellos von Michael Kern II geschaffen.

Über den Verstorbenen geben die Forchtenberger Kirchenregister Auskunft. Demnach stammt Jörg Zöller aus Weikersheim. Sein gleichnamiger Vater war bereits verstorben, als Zöller am 12. November 1583 in Forchtenberg Margarethe, die Tochter des Wolf Seyboldt, heiratete ${ }^{36}$. Aus der Ehe gingen den Taufregistern zufolge mindestens zehn Kinder hervor, von denen mindestens fünf bereits früh verstarben $^{37}$.

Schon etwas länger zurück liegen die Forschungsergebnisse von Anneliese Seeliger-Zeiss, der es gelungen ist, im Zuge der Bearbeitung der Ludwigsburger und

34 Jakob 1,12. Wahl des Bibelspruchs möglicherweise in Anspielung auf das Wappenbild.

35 Römer 14,7-8.

36 Alle Angaben nach Evangelisches Pfarrarchiv Forchtenberg Kirchenbuch 2, Eheregister zu 1583 (Landeskirchliches Archiv Stuttgart Film KB 1338).

37 Ev. Pfarrarchiv Forchtenberg Kirchenbuch 1, Taufregister und Totenregister (Landeskirchl. Archiv Stuttgart Film KB 1338): Barbara 1586 Dez. 12, Anna 1588 Jan. 14, Margaretha 1589 Sept. 22, Leonhard 1591 Jan. 3 (†1591 Mai 28), Gabriel 1592 Juli 19 (†1597 Aug. 8), Appollonia 1594 Sept. 6 (†1599 Sept. 18), Ammeley 1595 März 7, Zwillinge Petrus und Gabriel 1598 Aug. 1 (†1598 Aug. 1 bzw. Aug. 7), Jörg 1599 Juni 28. 
Böblinger Inschriften die Schriftmerkmale (Fraktur/Kapitalis) der Leonberger Bildhauerwerkstatt des Jeremias Schwartz und seiner Nachfolger („Leonberg II“) herauszuarbeiten und dadurch deren Werkkatalog, der auch Arbeiten in der Kurpfalz umfasst, beträchtlich zu erweitern ${ }^{38}$. Schwartz arbeitete sowohl für den Niederadel als auch für die „Ehrbarkeit“. Das Absatzgebiet der Werkstatt reichte, wie sich bei der Bearbeitung der Inschriften des Landkreises Göppingen zeigte, im Osten bis ins ulmische Herrschaftsgebiet ${ }^{39}$.

Eine andere große Werkgruppe, zu der ausschließlich Grabmäler des Niederadels aus dem zweiten Drittel des 16. Jahrhunderts gehören, bleibt dagegen nach wie vor anonym. Nach zwei Schwerpunkten ihrer Inschriftenproduktion im MainTauber-Kreis habe ich sie vorläufig mit dem Notnamen „Meister von Niederstetten und Wachbach" benannt ${ }^{40}$. Dieser Meister, dessen figürliche Darstellungen freilich nicht unbedingt als meisterlich zu bezeichnen sind, bediente sich einer mit Fremdformen durchsetzten Kapitalis und einer etwas ungelenk wirkenden Gotischen Minuskel mit streng begrenztem Repertoir an Versalien (Abb. 5). Insgesamt können diesem Steinmetzen 27 Arbeiten vorwiegend im ehemaligen Landkreis Mergentheim, im Hohenlohekreis und im Landkreis Schwäbisch Hall zugeschrieben werden, das Absatzgebiet reichte aber im Westen und Süden bis Schwaigern (Lkr. Heilbronn), Unterriexingen (Lkr. Ludwigsburg) und sogar bis Flehingen im Landkreis Karlsruhe.

Ganz neu „auf dem Radar“ befindet sich aktuell der aus Rot am See stammende und dort zunächst tätige Steinmetz Hans Weber, der sich 1621 in Crailsheim niederließ und im ersten Drittel des 17. Jahrhunderts eine große Zahl von Inschriftendenkmälern für brandenburg-ansbachische Diener, Amtleute und Pfarrer anfertigte $^{41}$, im Übrigen nach ersten Stichproben auch im mittelfränkischen Landkreis Ansbach ${ }^{42}$. Von ihm mit $H$ (ans) W(eber) S(teinmetz) signiert und archivalisch für ihn gesichert ist die Kanzel in Westgartshausen (Stadt Crailsheim) von 1611, von

${ }^{38}$ DI 47 (Böblingen, wie Anm.17) S.XLIII; - Anneliese Seeliger-Zeiss: Bietigheimer Grabmäler der Spätrenaissance aus der Werkstatt des Jeremias Schwartz aus Leonberg. In: Blätter zur Stadtgeschichte Bietigheim 7 (1988) S. 103-136; - Dies.: Heidelberger Werke des Bildhauers Jeremias Schwartz von Leonberg. In: Jahrbuch der Staatlichen Kunstsammlungen in Baden-Württemberg 29 (1992) S. 105-128; - Dies.: Leonberger Grabmäler des Bildhauers Jeremias Schwartz und seiner Nachfolger. In: Ein seliges end und fröhliche ufferstehung. Die Leonberger Grabmäler des Bildhauers Jeremias Schwartz in ihrer sozial- und kunstgeschichtlichen Bedeutung (Beiträge zur Stadtgeschichte 5). Leonberg 1998. S. 59-156.

${ }^{39}$ Grabplatte des Karl von Degenfeld $(\dagger 1575)$ an der katholischen Pfarrkirche Mariä Himmelfahrt in Eybach (Stadt Geislingen an der Steige); vgl. DI 41 (Göppingen, wie Anm. 17) Nr. 294 mit Abb. 122.

${ }^{40}$ DI 54 (Mergentheim, wie Anm. 18) S. LVIf., LX; DI 73 (Hohenlohekreis, wie Anm. 18) S. 61, 64 .

${ }^{41}$ Vgl. künftig Die Inschriften des Landkreises Schwäbisch Hall I. Ehemaliger Landkreis Crailsheim. Ges.u. bearb. von Harald Drös (Die Deutschen Inschriften; in Vorbereitung).

${ }^{42}$ So z.B. die Grabplatte des Franz Dieterich, markgräflich brandenburg-ansbachischen Vogts zu Feuchtwangen $(\dagger 1630)$ an der Friedhofskapelle in Feuchtwangen und das Epitaph 


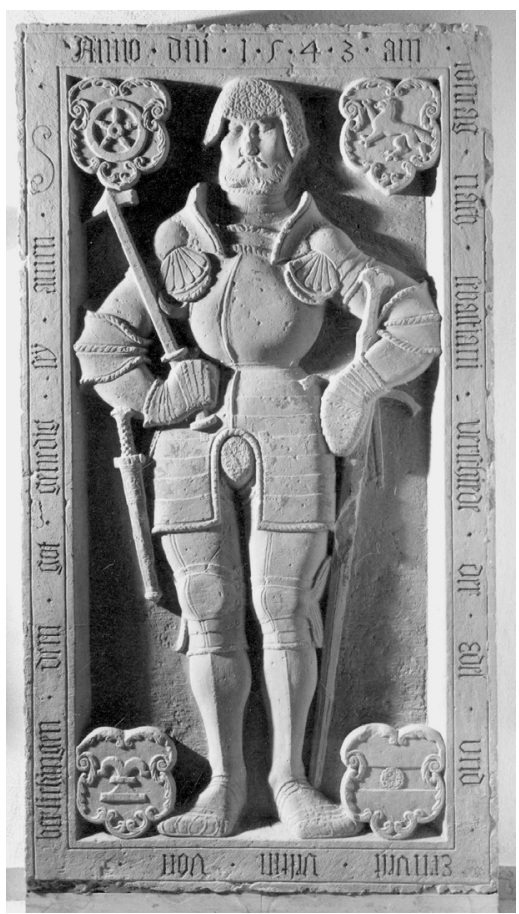

Abb. 5: „Meister von Niederstetten und Wachbach“, Epitaph des Valentin von Berlichingen (1543) in Dörzbach (Hohenlohekreis).

der ausgehend das bislang völlig unbeachtete beträchtliche Oeuvre rekonstruiert werden kann, zu dessen frühesten Erzeugnissen der Taufstein von 1606 in der Pfarrkirche in Rot am See zählt. Auch dieser ist mit $H W$ signiert und trägt dasselbe Steinmetzzeichen wie die Kanzel in Westgartshausen. Eine „Ohrmarke“ von Webers Kapitalis ist ein C, dessen beide Bogenenden ohne Sporen spitz zulaufen (Abb.6). Seine Fraktur ist ebenfalls leicht zu erkennen. Das o ist gestreckt-sechseckig und liefert die Grundform für die übrigen Bögen der Gemeinen. Die Versalien sind zumeist schlicht und wenig verziert, sie wirken jedoch mitunter unproportioniert, so besonders $A$ und $F$, die zumeist deutlich unter die Grundlinie reichen. Ein weiteres Merkmal, das Weber im Übrigen mit der Forchtenberger Kern-Werkstatt gemein hat, ist seine Vorliebe für das Material Alabaster, vor allem für Kindergrabplatten.

des Christoph Sebastian von Jaxtheim $(\dagger 1633)$ in der ev. Pfarrkirche St. Gumpertus in Ansbach. 


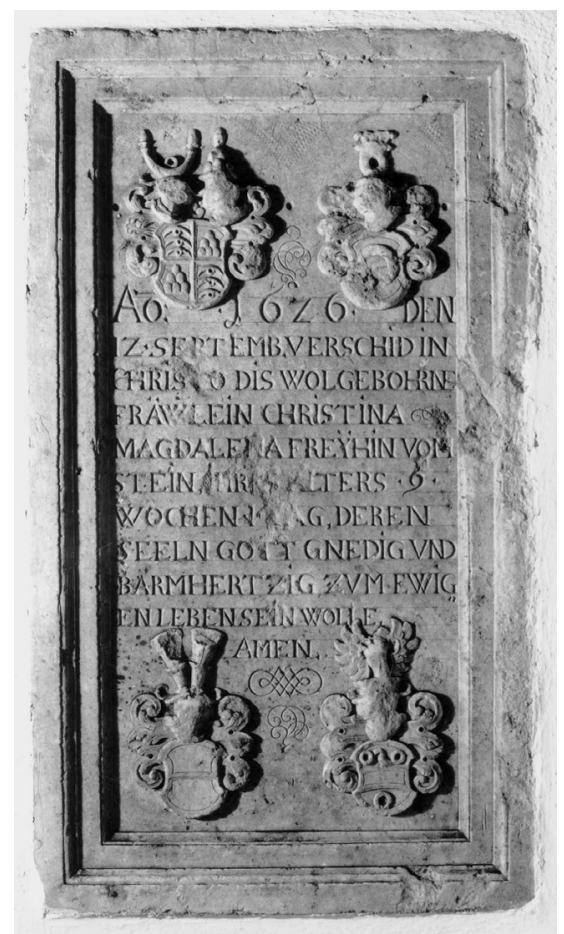

Abb. 6: Hans Weber, Grabplatte der Christina Magdalena vom Stain (1626) in Wildenstein (Gde. Fichtenau, Lkr. Schwäbisch Hall).

Auf Bölgentaler Gemarkung nordwestlich von Gröningen (Landkreis Schwäbisch Hall) steht die sogenannte „Anhäuser Mauer“, der Rest des Pauliner-Eremitenklosters Anhausen. In die einsam im freien Feld aufragende Mauer der ehemaligen Klosterkirche ist eine Reihe von fünf Denkmälern der Herren von Bebenburg eingelassen, die alle vermutlich gleichzeitig gegen Ende des 15. Jahrhunderts, vielleicht um 1481 gefertigt worden sind. Ohne näher auf die Probleme der genaueren zeitlichen Einordnung und der Inschriftentranskription einzugehen ${ }^{43}$ sei lediglich auf das Ergebnis des Schriftvergleichs hingewiesen, der ergab, dass die Denkmälerserie - was bisher nicht bekannt war - von demselben Meister gehauen wurde, der gemeinhin als der „Hauptmeister der Ansbacher Schwanenordensritter“ bekannt ist und der zahlreiche Grabmäler für Angehörige des brandenburg-ansbachischen Schwanenordens im heutigen Landkreis Ansbach geschaffen hat ${ }^{44}$. Die Anhäuser Denkmäler könnten sogar eines seiner frühesten Werke sein.

${ }^{43}$ Vgl. dazu künftig DI Schwäbisch Hall I (wie Anm. 41).

${ }^{44} \mathrm{Zu}$ diesem vgl. u.a. Wilhelm Funk: Der Hauptmeister der Ansbacher Schwanenordens- 
Weitere Werkstätten, die man allein anhand ihrer epigraphischen Merkmale eindeutig erkennen kann, ließen sich aufzählen, so ein vermutlich in Öhringen ansässiger Steinmetz mit einer charakteristischen, dekorativen, bewusst altertümlich verfremdeten Kapitalis, von dem acht kurz nach der Mitte des 16. Jahrhunderts entstandene Inschriftenträger im Hohenlohekreis geschaffen wurden, darunter auch das Epitaph für Götz von Berlichingen mit der eisernen Hand im Kloster Schöntal sowie eine Bauinschrift an dessen Schloss in Rossach ${ }^{45}$. Sodann die Ulmer Werkstätten der Bildhauer Hans $(\dagger 1594)$ und Michael Schaller (1613 †), Peter Schmid (†1608) und Georg Huber (†zwischen 1628 und 1648) mit ihrer ungewöhnlich „langlebigen“ Gotischen Minuskel ${ }^{46}$, deren Absatzmarkt weit ins bayerische Schwaben hineinreichte ${ }^{47}$.

Im Zuge der Bearbeitung der Schwäbisch Haller Inschriften sollte es auch gelingen, das umfangreiche Oeuvre des aus Laudenbach bei Weikersheim stammenden Haller Bildhauers Sem Schlör $(† 1597 / 98)$ und seiner produktiven Werkstatt epigraphisch genauer zu bestimmen als das bisher der Fall war. Als offensichtlich eigenhändige Frühwerke Schlörs, für die er eine vom klassischen Kanon weit entfernte rustikale Kapitalis verwendete, können schon jetzt zahlreiche in den 1550 er Jahren entstandene Arbeiten identifiziert werden, darunter als bislang frühestes bekanntes Werk die 1552 entstandene Grabplatte der Gräfin Helena von Hohenlohe (Abb. 7) in Kloster Gnadental (Landkreis Schwäbisch Hall) ${ }^{48}$.

Neben der Ermittlung von Werkstattzusammenhängen dient die Inschriftenpaläographie auch einer zunehmenden Verfeinerung des Datierungsrasters und liefert somit einen Beitrag zur allgemeinen Schriftgeschichte. Es geht darum, festzustellen, welche Schriftphänomene wann erstmals auftreten, wann sie sich verändern, wann sie verschwinden. Somit wird eine immer genauere zeitliche Einordnung undatierter oder nur mehr fragmentarisch erhaltener Inschriften ermöglicht. Ein Beispiel soll genügen: Die sogenannte Frühhumanistische Kapitalis verdankt ihre Entstehung Bestrebungen humanistischer Kreise, in der Orientierung an klassischen oder besser an „vorgotischen“ Vorbildern wieder klar lesbare Schriften zu erzielen. In dieser Schriftart fließen Elemente der Kapitalis und vorgotischer Schriften vornehmlich des 12. und 13. Jahrhunderts sowie vereinzelt griechisch-byzantinische Schrifteigentümlichkeiten zusammen. Die Schrift wurde zuerst in Süddeutschland ab der Mitte und verstärkt im letzten Viertel des 15. Jahrhunderts in breiterem Umfang auch für Inschriften verwendet. Eine vermittelnde Funktion kam den humanistischen Zentren Süddeutschlands wie den Konzilsorten Kon-

ritter. In: Neue Beiträge zur allgemeinen und fränkischen Kunstgeschichte in Vergangenheit und Gegenwart. FS 1904-1964. Kunstverein Erlangen. Erlangen 1965. S. 136-146.

${ }^{45}$ Vgl. DI 73 (Hohenlohekreis, wie Anm. 18) S. 66.

46 Vgl. DI 41 (Göppingen, wie Anm. 17) S. LIf., LIV.

47 Vgl. dazu etwa Die Inschriften des Landkreises Günzburg. Ges.u. bearb. von Claudia Madel-Böbringer (DI 44). Wiesbaden 1997. S. XXVIf., XXX.

48 Vgl. u.a. DI 73 (Hohenlohekreis, wie Anm. 18) S. 67, Nr. 280. 


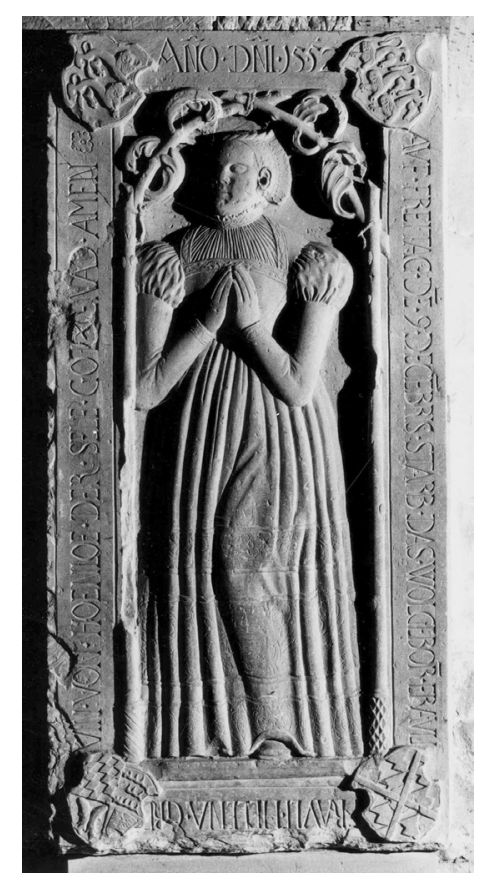

Abb. 7: Sem Schlör, Grabplatte der Gräfin Helena von Hohenlohe (1552) in Gnadental (Gde. Michelfeld, Lkr. Schwäbisch Hall).

stanz und Basel zu. Ein frühes Zentrum inschriftlicher Umsetzung der Schrift war ferner die Residenzstadt Kaiser Friedrichs III. Wiener Neustadt, wo sie ab 1442 vorkommt und besonders häufig als Schrift für Friedrichs Devise AEIOV Verwendung fand ${ }^{49}$. Andernorts fand die Schrift jedoch zunächst nur wenig Resonanz. Die dekorative Wirkung und der Charakter einer Auszeichnungsschrift prädestinierte die Frühhumanistische Kapitalis zur Verwendung in der Buch- und Tafelmalerei, etwa für Spruchbänder, Nimben- und Gewandsauminschriften, und im Kunsthandwerk, wo sie in den meisten süddeutschen Regionen freilich erst ab dem letzten Viertel des 15. Jahrhunderts in den inschriftlichen Bereich eindringt. Erinnert sei als eines der prominentesten Beispiele an die Devise ATTEMPTO des Grafen Eberhard im Bart, die sich auch in inschriftlicher Umsetzung (neben einer Ausführung in Gotischer Minuskel) häufig findet ${ }^{50}$.

49 Die Inschriften des Bundeslandes Niederösterreich. Tl.2: Die Inschriften der Stadt Wiener Neustadt. Ges.u. bearb. von Renate Kobn (DI 48). Wien 1998. S. XLVI-XLVIII, LVIII-LXI.

${ }^{50}$ So etwa auf der Grabplatte Herzog Eberhards $(\dagger 1496)$ in der Tübinger Stiftskirche (vgl. Harald Schukraft: Die Grablegen des Hauses Württemberg. Stuttgart 1989. Taf. 2) und auf 
Vor diesem Befund verdient das erste Auftreten der Schriftart im Hohenlohekreis auf der wohl in den 60er Jahren des 15 . Jahrhunderts entstandenen sogenannten "Stiftertumba“ in der Öhringer Stiftskirche Beachtung ${ }^{51}$ (Abb. 8). Die Schrift wurde hier offenbar bewusst gewählt, um den Eindruck ehrwürdigen Alters zu erzielen. Bei der Suche nach ähnlich frühen in dieser Schriftart ausgeführten Inschriften in der Region stößt man auf zwei Nameneinträge - wohl Grabbezeugungen - von 1467 an der Pfarrkirche in Michelbach an der Bilz (Landkreis Schwäbisch Hall) und auf Grabmäler der Grafen von Hohenlohe und der Schenken von Limpurg in Gnadental und Großkomburg. Jedenfalls zeigt das Öhringer Beispiel, dass man auch in nördlichen Regionen Süddeutschlands durchaus mit früherem Vorkommen der Frühhumanistischen Kapitalis rechnen darf als bislang angenommen.

Die Inschriftenpaläographie als genuin epigraphische Methode erbringt in der Regel am ehesten neue Forschungsergebnisse, weshalb sie hier auch vorrangig und ausführlicher vorgestellt wurde. Weniger spektakulär sind dagegen die - in einzelnen Bearbeitungsgebieten gleichwohl bisweilen recht umfangreichen - Neufunde, seien es bisher unbeachtete Originale oder seien es kopial überlieferte Inschriften in Archiven und Bibliotheken. Häufig sind es Zufallsfunde, und leider gelingen sie nicht selten erst dann, wenn ein Inschriftenband bereits abgeschlossen und publiziert ist, wie dies bei dem oben aufgeführten Forchtenberger Beispiel der Fall war.

Ein weiterer Nachtrag, diesmal aufgrund eines Neufunds im Archiv, betrifft den Rems-Murr-Kreis: In der ehemaligen Schlosskirche in Winnenden steht das von dem Heilbronner Bildhauer Jakob Müller geschaffene Epitaph des 1608 gestorbenen Deutschordenskomturs Johann von Gleichen. Es zeigt den Verstorbenen im Harnisch, auf einem Löwen stehend. Auf den flankierenden Säulen sind die Wappen einer 16-Ahnenprobe mit Beischriften angebracht. Der Sockel ist heute leer. Er enthielt ursprünglich zwei Schrifttafeln aus Schiefer mit vergoldeter Schrift. Die rechte Tafel war schon längere Zeit verloren, die linke war zuletzt bis zum Kirchenumbau von 1980 lose beiseite gestellt und ist seither ebenfalls verschollen. Sie ist auf einem Foto des Landesdenkmalamts noch zu sehen, die Inschrift ist wegen des winzigen Formats freilich nur mehr mit Mühe zu entziffern ${ }^{52}$. Eine ältere Textwiedergabe von 1923 ist zu ungenau und zudem verkürzend, um bei der Lesung zu helfen. Der Text beider Tafeln war fortlaufend in durchgehenden Zeilen zu lesen, so dass der zuletzt erhaltene Text jeweils nur die ersten Hälften der acht Zeilen enthielt. Eine befriedigende Textergänzung war bislang nicht möglich, im DI-Band Rems-Murr-Kreis wurde folglich nur der halbe Text des Sterbevermerks geboten ${ }^{53}$.

Im Hohenlohe-Zentralarchiv Neuenstein fand ich nun kürzlich im Nachlass des fürstlich hohenlohischen Domänendirektors und Archivars Joseph Albrecht

der Wappentafel von 1491 am Beinsteiner Torturm in Waiblingen; vgl. DI 37 (Rems-MurrKreis, wie Anm. 17) Nr. 57 mit Abb. 33.

51 DI 73 (Hohenlohekreis, wie Anm. 18) Nr. 75.

52 Abb. in Die Kunstdenkmäler des Rems-Murr-Kreises 2 (wie Anm. 5) S. 1546.

${ }^{53}$ DI 37 (Rems-Murr-Kreis, wie Anm. 17) Nr. 256. 


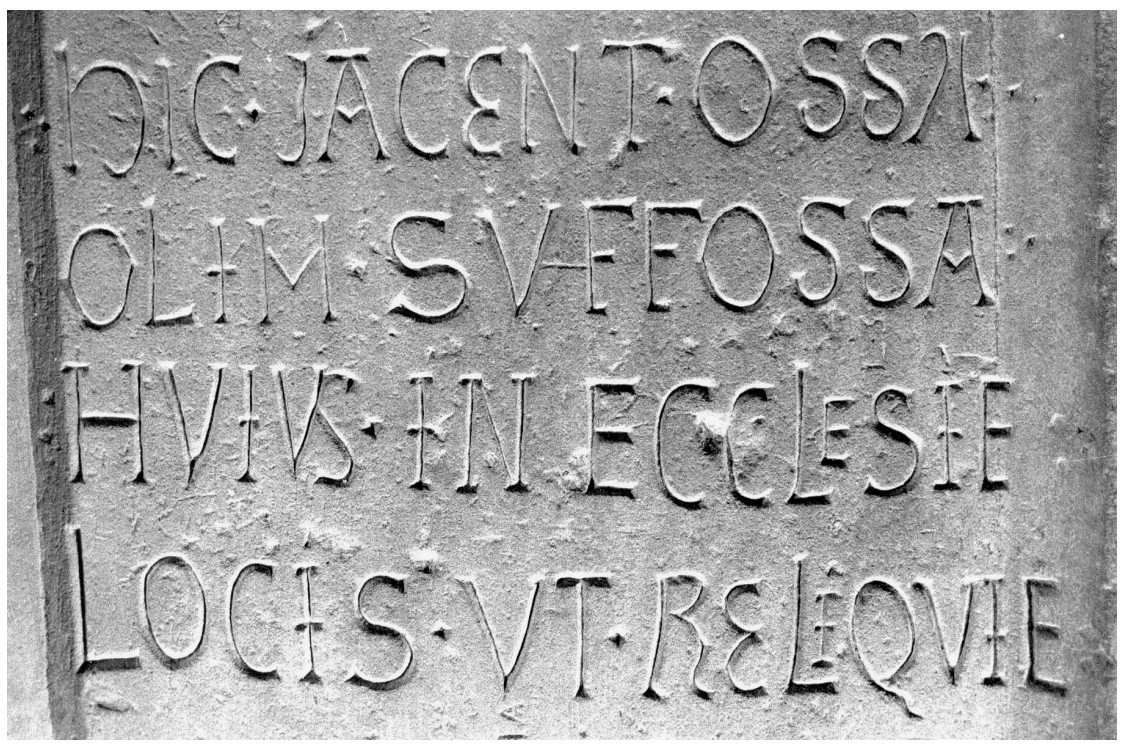

Abb. 8: Öhringen (Hohenlohekreis), Stiftertumba (um 1460-70).

(1803-1871) eine 1843 angefertigte Beschreibung und Abzeichnung des Epitaphs mit einer Transkription der vollständigen Inschrift. Schloss Winnenthal wurde damals als Heilanstalt genutzt, und die Beschreibung des Epitaphs verdanken wir dem aus Langenburg stammenden Drechsler und Aufseher der Anstalt Johann Lü$\operatorname{der}^{54}$. Kurioserweise hatte Albrecht sich für das Grabmal interessiert, weil er einen Zusammenhang mit den Grafen von Gleichen vermutete, über die er Nachforschungen anstellte im Zusammenhang mit seinen Arbeiten zur Geschichte des Hauses Hohenlohe. Die Grafen von Hohenlohe aus der Neuensteiner Linie hatten 1631 nach dem Aussterben der Grafen von Gleichen die sogenannte Obergrafschaft geerbt und führten seither auch den Titel und das Wappen der Grafen von Gleichen. So hat sich aufgrund eines Irrtums Albrechts die Inschrift Johanns von Gleichen erhalten, der gar nicht dem Grafengeschlecht, sondern einem thüringischen Niederadelsgeschlecht dieses Namens angehörte. Bei einer gezielten Suche nach Kopialüberlieferungen der Winnender Inschrift wäre man sicherlich nie auf diesen Fund gestoßen. Doch so lässt sich der Sterbevermerk des Grabmals jetzt vollständig edieren, wobei sich auch geringfügige Änderungen gegenüber dem Text in DI

${ }^{44}$ Hohenlohe-Zentralarchiv Neuenstein GA 55 (Nachlaß Albrecht) Bü 229, darin: Beschreibung des Leichensteins des Herrn von Gleichen in der Schloß-Kirche zu Winnenthal, von Johann Lüder aus Langenburg, Drechsler u. Aufseher in der Kgl. Heilanstalt Winnenthal, 1843 IV 12. Die Textwiedergabe ist, wie ein Vergleich mit dem auf dem Foto noch lesbaren Inschriftenteil zeigt, nicht immer buchstaben-, wohl aber wortgetreu. 
Rems-Murr-Kreis ergeben (der nach der Abschrift im Neuensteiner Archiv ergänzte Teil der Inschrift hier kursiv gesetzt):

Anno Domini 1608 den 6 Februarij abents // umb 9 Ubr verschied in Gott seeliglich der Ehrwürdige, Edel, // Veste vnd Gestreng Herr Johan von Gleichen Teutsch // Ordens ritter, der Bolley Franken, Rathsgebietiger // vnd Comenthur $\mathrm{zu}$ Wienenden · vnd wurde den // $12^{\text {ten }}$ dieszes, in diesem Gottes=Hause christlich begraben, // seines alltters 81 Jhar der Allmechtig Gott wolle // Jhme an seinem groszen Tage mit allen // auszerwelten ein Fröliche vfferstehung vns // Allen aber zu seiner Zeit eine seelige Sterbestündlein // verleihen Amen. Deszen zu Christ seliger gedecht//nisz und sonderbaren Ebren haben die auch Edeln, // Gestrengen vnd vesten. seine freundliche liebe Ve//ttern, Christoph und Hanns Heinrich, Vatter und // Sobn von Beringen zu Pattichendorf vff Schieben vnd wei//ndlitss, Jhme das Epitaphium machen und aufrichten laszen.

Herausragende Neufunde von Originalinschriften sind hingegen eher selten. Für den badischen Landesteil sei an die spektakulären Grabungen im Bereich der Schönauer Klosterkirche vor knapp 20 Jahren erinnert, bei denen etliche mittelalterliche Grabplatten entdeckt wurden ${ }^{55}$.

Ein aufregender Fund wurde uns aber im Sommer 2011 aus Maulbronn gemeldet. Der Steinheimer Heimatpfleger Hans Dietl informierte uns freundlicherweise darüber, daß in der Mauer eines ehemaligen Weinbergs in unmittelbarer Nähe des Klosters kürzlich eine Inschrift vom Bewuchs befreit und freigelegt worden war (Abb. 9). Die Transkription der Inschrift lautet:

Anno Mccccxcvij . B(ruder). Hans Zatz Von /

Zeysenhusen Vogtbruder . hat disen Wingart /

gemacht . All hernach · Lieben Brieder .

Hans Zatz, der hier als „Bruder“ bezeichnet ist, muss demnach ein Maulbronner Mönch (oder Konverse?) gewesen sein. Was es mit dem Titel „Vogtbruder“ auf sich hat, konnte noch nicht geklärt werden. Der aus Zaisenhausen stammende Hans Zatz ist übrigens in einer zweiten Inschrift dokumentiert, die ebenfalls 1497 und nach Ausweis der weitgehend identischen Schriftformen auch sicherlich von dem selben Steinmetzen gefertigt wurde. Es könnte sich bei dem Inschriftenträger, der in der Erdgeschoßhalle des Maulbronner Ephorats aufgestellt ist, um den Sockel eines Kruzifixus gehandelt haben (Abb. 10). Die Lesung dieser Inschrift, die im DI-Band Enzkreis von Renate Neumüllers-Klauser ediert wurde ${ }^{56}$, lässt sich nach

55 Vgl. Harald Drös: Sechs neu aufgefundene mittelalterliche Grabplatten in der Kirche der ehemaligen Zisterzienserabtei Schönau, Rhein-Neckar-Kreis. In: Fundberichte aus Baden-Württemberg 23 (2000) S. 629-652.

56 DI 22 (Enzkreis, wie Anm. 16) Nr. 127: B. Hanns / Zar; / 1497. 


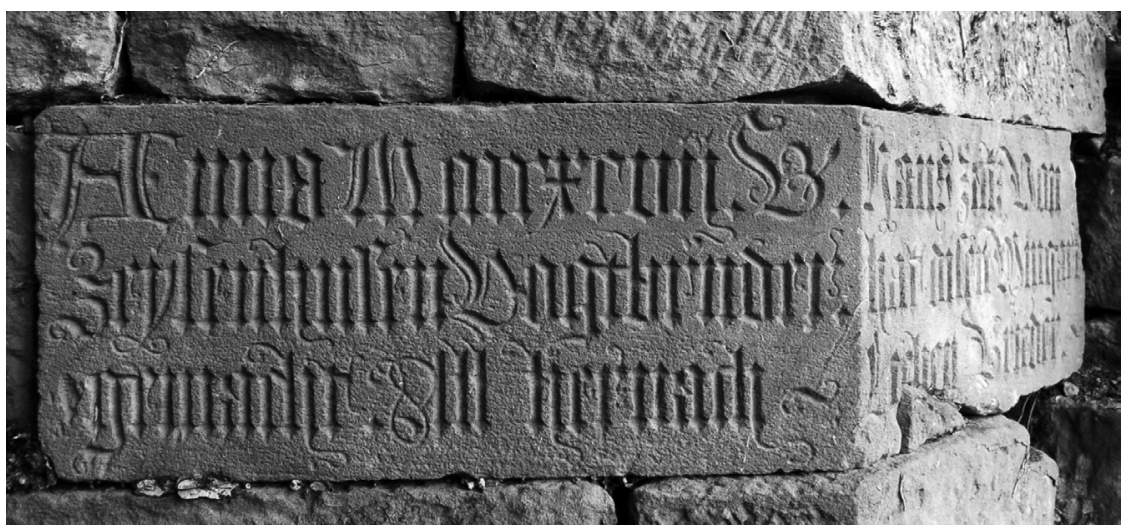

Abb. 9: Maulbronn (Enzkreis), Wingertinschrift des Hans Zatz (1497).

Kenntnis der neu aufgefundenen Inschrift nunmehr korrigieren zu: B(ruder) Hanns / Zatz / 1497

„Gemacht“ ist in der Wingert-Inschrift vermutlich in der Bedeutung von „in Handarbeit angelegt“ zu verstehen, andernfalls müsste man „gestiftet“, „vermacht“ o. ä. erwarten. „All hernach“ ist eine auch anderweitig in Inschriften, zumeist in Grabinschriften, vorkommende Devise, die auf die Vergänglichkeit hinweist ${ }^{57}$. Mit diesem Spruch wendet sich Zatz nach Ausweis der abschließenden Wörter der Inschrift an seine Mitbrüder. Der Neufund ist jedenfalls aufgrund seines ungewöhnlichen Formulars und seiner hervorragenden, kunstvollen Schriftgestaltung der Gotischen Minuskel und ihrer Versalien von einiger Bedeutung.

Abschließend möchte ich noch an zwei Beispielen aus dem Hohenlohekreis zeigen, wie wichtig es ist, Inschriften flächendeckend vollständig zu erfassen, um so erst durch das Erkennen und Herstellen von Querverbindungen offene Fragen einer Lösung näher zu bringen.

Die Grabplatte des 1551 verstorbenen Grafen Georg I. von Hohenlohe-Waldenburg in der Stadtkirche in Waldenburg ${ }^{58}$ ist mit Metallauflagen geschmückt: Im Feld das große linksgewendete Vollwappen der Grafen von Hohenlohe, in den vier Ecken kleinere Ahnenwappen. Die in Gotischer Minuskel erhaben gegossene Umschrift lautet:

$57 \mathrm{Vgl}$. etwa das Epitaph des Administrators des Hochmeistertums in Preußen und Deutschmeisters Walter von Kronberg $(\dagger 1543)$ in der katholischen Pfarrkirche St. Marien in Bad Mergentheim: DI 54 (Mergentheim, wie Anm.18) Nr.139. - Ferner allgemein: Petrus Ortmayr: „Allhernach“. Bedeutung und Verbreitung dieses Wortes auf oberösterreichischen Grabdenkmälern des 16. Jahrhunderts. In: Christliche Kunstblätter 90 (1952) S. 17-21.

${ }^{58}$ DI 73 (Hohenlohekreis, wie Anm. 18) Nr. 270 mit Abb. 148. 


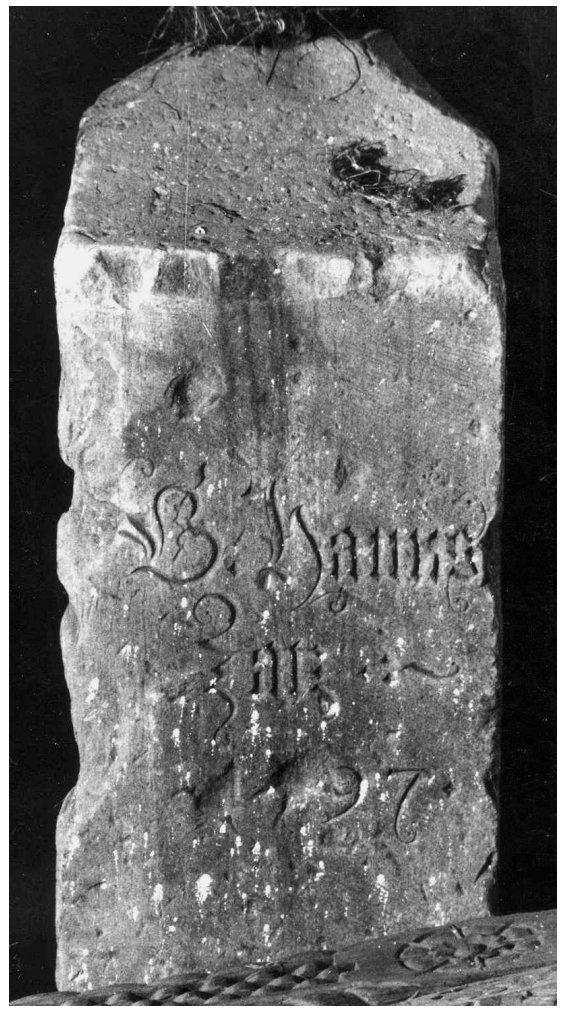

Abb. 10: Maulbronn (Enzkreis), Kruzifixsockel? (1497).

Anno Do(mi)nj · 1551 am montag nach dem Sontag / Judica der da was der · 16 • tag des monats Marcij starb der Wolgeborne Herr / Herr Jorig graff von Hohenloe $\cdot($ et $)$ c(etera $) a) \cdot$ des sele der /

und dann geht es auf der linken Randleiste weiter:

Sele der Almechtig ewig Gott gnedig vnd Barmhertzig sein Wöll

Hier ist offensichtlich etwas nicht in Ordnung. Der Sterbevermerk mit seinen Bestandteilen - Name und Sterbedatum - ist korrekt, doch in der abschließenden Fürbittformel kommen zwei Wörter („sele der“) doppelt vor. Bei genauerem Hinsehen stellt man schnell fest, dass dieser Fehler genau an der Übergangsstelle von der Fußleiste auf die linke Randleiste passiert ist (Abb.11). Und vergleicht man die 


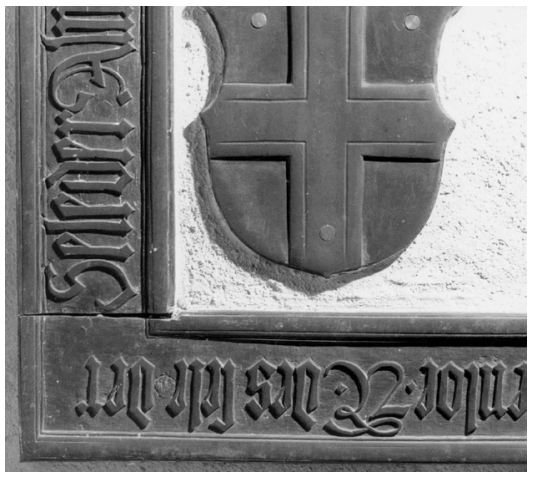

Abb. 11: Waldenburg (Hohenlohekreis), Grabplatte des Grafen Georg I. von Hohenlohe-Waldenburg (1551).

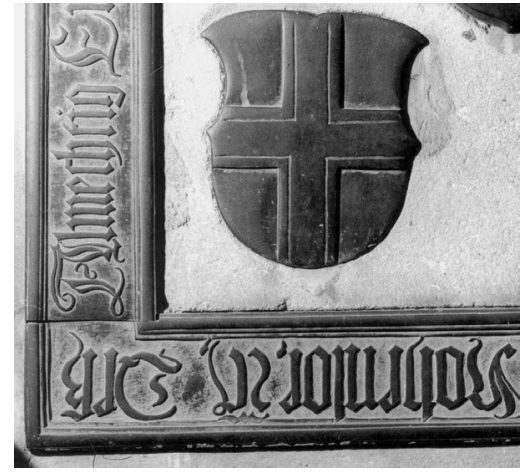

Abb. 12: Neuenstein (Hohenlohekreis), Grabplatte des Grafen Albrecht III. von Hohenlohe (1551).

Schrift, wird auch deutlich, dass an dieser Stelle ein Wechsel der Buchstabenformen, der Proportionen und der Buchstabenhöhe erfolgt. Wie ist dies zu erklären?

Die Lösung des Rätsels findet sich in Neuenstein: Dort befindet sich nämlich die Grabplatte Graf Albrechts III. von Hohenlohe, der im selben Jahr wie Georg verstorben ist ${ }^{59}$. Seine Grabplatte zeigt eine identische Gestaltung. Ganz offensichtlich wurden die Messingauflagen in derselben - Nürnberger - Werkstatt hergestellt wie die der Waldenburger Grabplatte. Für den Guß der Wappen wurden sogar dieselben Model benutzt. Die Inschrift lautet hier:

Anno Do(mi)nj; 1551 ; am tag Sewaldij der da / was der , 19 tag des Monats Augustj starb der Wolgeborne Herr herr / Albrecht Graff von Hohenloe , (et)c(etera), Desz /

und dann geht es weiter auf der linken Rahmenleiste:

Almechtig Ewig Gott gnedig vnnd Barmhertzig sein woell Amen •

Die Worte also, die hier fehlen („Seele der“) sind genau die, die auf der Waldenburger Grabplatte doppelt vorkommen. Und der Schriftbefund bestätigt den Verdacht (Abb. 12): auch hier ist ein Schriftwechsel an besagter Stelle festzustellen, nur in umgekehrter Richtung. Es ist also eindeutig, dass jeweils die linken Randleisten der beiden Grabplatten miteinander vertauscht wurden. Offenbar wurde der Guss der Metallteile für die beiden Grabplatten der kurz hintereinander verstorbenen

59 Ebd. Nr. 271 mit Abb. 149. 
Grafen gleichzeitig ausgeführt. Die Vertauschung der in der Folge dann falsch montierten Inschriftenleisten muss dann entweder bereits in der Werkstatt oder auf dem gemeinsamen Transport von Nürnberg in die Grafschaft Hohenlohe passiert sein. Bei genauer Untersuchung der Texte und Schriftformen der Inschriften lassen sich also gelegentlich Einblicke in die Art und Weise gewinnen, wie die Werkstätten gearbeitet haben: mitunter eben auch recht nachlässig.

Das zweite Beispiel führt zunächst nach Künzelsau. In den mittleren Fachwerkständer der Giebelfassade des Hauses Keltergasse 47 ist eine Inschrift in einer recht unbeholfenen Fraktur eingeschnitzt und nachträglich mit weißer Farbe ausgemalt worden, wobei allerdings einige Stellen falsch und die letzte Zeile gar nicht nachgezogen wurden ${ }^{60}$. Die Inschrift lautet:

$\cdot 1 \cdot 5 \cdot 9 \cdot 7 /$ Michel FrichKorn / Albrecht Franch / B $\cdot W \cdot M / \cdot V \cdot W /$

Hans Wegele $\cdot$ Anna Wegeleren

Während die beiden letzten Namen sicherlich den Bauherrn und seine Ehefrau bezeichnen, sind die Initialen auf die beiden ersten Namen zu beziehen und nach vergleichbarem Formular anderer Bauinschriften aufzulösen als:

$$
\mathrm{B}(\text { eide }) \cdot \mathrm{W}(\text { erk }) \cdot \mathrm{M}(\text { eister }) / \cdot \mathrm{V}(\text { on }) \cdot \mathrm{W}
$$

Für den Ortsnamen war freilich zunächst keine sichere Auflösung möglich, denn außer Weikersheim und Waldenburg gibt es in der näheren Umgebung Künzelsaus natürlich zahlreiche weitere Orte, die mit W beginnen.

Eine unerwartete Lösung des Problems brachte dann die Erfassung der Inschriften in der Stadt Öhringen. An dem dortigen von Bürgermeister Seebach 1602 erbauten stattlichen Fachwerkhaus Marktstraße 18 befinden sich gleich vier in die Fachwerkpfosten eingeschnitzte, zuvor in der Literatur nur sehr ungenau wiedergegebene Bauinschriften und Bauhandwerkernennungen ${ }^{61}$. Die ausführlichste nennt den Zimmermann und seine Gehilfen:

ANNO / 1602 / HADT / MICHAEL / FRISCH/KORN / BVRGER / VNND / ZIMMER/MAN ZV / WALDEN/BVRG / MIDT / SEINEN / GESELN / BARTEL / MEISNER / ERHART / RETER / CLAVS / SCHRAM / HANS / KRAFT / DISEN / BAV VER/FERTIGT / DEN · 21 / IVLIJ VF/GERICHT

Hier begegnet also wieder Michael Frischkorn, als Herkunftsbezeichnung in der Künzelsauer Inschrift ist demnach Waldenburg zu ergänzen. Und dass auch das Öhringer Haus wieder eine Gemeinschaftsarbeit der beiden Werkmeister war,

60 Ebd. Nr. 495.

61 Ebd. Nr. 561. 
zeigt eine zweite dort angebrachte Inschrift, die nun wiederum ohne Kenntnis der Künzelsauer Inschrift unverständlich bliebe. Sie ist aufzulösen als:

$\cdot 1 \cdot 60 \cdot 2 \cdot / \mathrm{M}($ ichael $) \cdot \mathrm{F}($ rischkorn $) \cdot \mathrm{A}($ lbrecht $) \cdot \mathrm{F}($ ranck $)$

Die gezeigten Beispiele mögen genügen, um die Bedeutung und den besonderen Wert einer in der Fläche kontinuierlich voranschreitenden Edition inschriftlicher Quellen aufzuzeigen. Eine Beschränkung auf vermeintliche inschriftliche Schwerpunkte und kulturelle Zentren und ein Aussparen der weiten ländlichen Umgebung würde den Blick auf die Zusammenhänge, wie sie hier exemplarisch vorgestellt wurden, hingegen verbauen. Die Heidelberger Inschriftenkommission wird daher auch künftig an diesem bewährten Konzept festhalten.

\section{Abildungsnachweise:}

Abb. 1-3, 6-8, 10-12: Inschriftenkommission der Heidelberger Akademie der Wissenschaften (Foto Harald Drös).

Abb. 4: Harald Drös.

Abb. 5: Dr. Helmut Hartmann, Bechtheim.

Abb. 9: Hans Dietl, Steinheim an der Murr. 